\title{
Intrathecal methotrexate combination with systemic chemotherapy in glioblastoma patients with leptomeningeal metastases
}

xun kang ( $\sim$ kangx1987@sina.com )

Beijing Tiantan Hospital https://orcid.org/0000-0002-8940-8402

\section{Feng Chen}

Beijing Tiantan Hospital

\section{Zhuang Kang}

Beijing Tiantan Hospital

\section{Ce Wang}

Beijing Tiantan Hospital

Shoubo Yang

Beijing Tiantan Hospital

\section{Yali Wang}

Beijing Tiantan Hospital

\section{Zenghui Qian}

Beijing Tiantan Hospital

\section{Yan Li}

Beijing Shijitan Hospital

\section{Shan Li}

Beijing Shijitan Hospital Jianxin Chen

Beijing Shijitan Hospital

Hao Lin

Beijing Tiantan Hospital

Aizhong Fang

Beijing Tiantan Hospital

Yi Lin

Beijing Tiantan Hospital

\section{Bo Jiang}

Beijing Tiantan Hospital

\section{Parker Li}

Shanghai Jiao Tong University Medical Library: Shanghai Jiao Tong University School of Medicine Yichen Peng 
Beijing Tiantan Hospital

\section{Xiaomin Wang}

Beijing Tiantan Hospital

Wenbin Li

Beijing Tiantan Hospital

\section{Research Article}

Keywords: Glioma, Glioblastoma, Leptomeningeal disease, Intrathecal methotrexate

Posted Date: April 26th, 2021

DOI: https://doi.org/10.21203/rs.3.rs-452592/v1

License: (c) (1) This work is licensed under a Creative Commons Attribution 4.0 International License. Read Full License 


\section{Abstract \\ Purpose}

Glioblastoma (GBM) is the most common and aggressive primary malignant brain tumor with a bad outcome. Leptomeningeal disease (LMD) is a severe complication of GBM, with a worse outcome and have no effective treatment. A combination chemotherapy was conducted to evaluated the effective of GBM patients with LMD.

\section{Methods}

A retrospective analysis was conducted of GBM patients diagnosed with LMD between January 2012 and December 2019 in our institution. All these patients accepted at least one cycle of combination therapy combination intrathecal methotrexate(MTX) with systemic chemotherapy. Clinical and pathological data were analyzed to explore the effective treatment and the outcome of GBM patients with LMD.

\section{Results}

Twenty-six patients were enrolled in this study. The median time from GBM diagnosis to LMD development was 9.3 months (range 2-59 months). The median overall survival of LMD patients from diagnosis after accepted systemic chemotherapy combination with intrathecal methotrexate was 10.5 months (range 2-59 months). In the Cox univariate analysis, gross resection of tumor $(p=0.022)$, KPS $\varangle 60(p=0.002)$ and Ommaya reservoir implantation ( $p \otimes 0.001)$ were correlated with survival. Multivariate analysis showed that KPS $₫ 60(p=0.037)$ and Ommaya reservoir implantation $(p=0.014)$ were positive factors correlated with survival. The common toxicities of combination therapy were myelotoxicity and gastrointestinal reactions. According to Common Terminology Criteria of Adverse Events 4.03 , most of the toxicities were less than grade 3 .

\section{Conclusion}

Intrathecal methotrexate combined with systemic chemotherapy is an effective treatment for GBM patients with LMD and has mild treatment-related side effects.

\section{Introduction}

Glioblastoma (GBM) is one of the most malignant brain tumor with a median overall survival (OS) of 14.6 months even with surgery, radiotherapy, and temozolomide (TMZ) treatment $[1,2]$. Although the survival time of GBM has increased to 20.9 months after treatment with a combination of tumor-treating fields (TTF), it was still limited[3]. Leptomeningeal spread of disease (LMD) is known as glioma cell invasion into the cerebrospinal fluid (CSF) or leptomeninges. GBM patients with LMD often showed worse 
prognosis than the progression of parenchymal disease and had a median survival of 2-5 months[4-6]. LMD was initially considered a rare complication of gliomas, but the incidence of LMD seems to be higher than the estimated rate of $4 \%$, reaching $25 \%$ in postmortem neuropathological studies $[4,7,8]$. Recent studies indicated that LMD incidence is increasing, possibly because of improvement in survival rates and survival times[9, 10]. Diagnosis of LMD involves enhanced magnetic resonance imaging (MRI) and/or positive morphology of CSF cells.

Intrathecal injection of MTX is an effective method for treating glioma with $L M D$, and our team found that MTX can inhibit the growth of GBM cells by downregulating Ras/MAPK/myc/CD47 signaling pathway[11]. However, MTX intrathecal injection is insufficient, because it is ineffective on tumor cells in the brain parenchyma. Thus, a combined systemic treatment is needed. TMZ and its combination regimen and etoposide combined with platinum chemotherapy regimen are optional systemic treatments. We used MTX intrathecal combination with systemic chemotherapy as a method for patients with LMD.

This study aims to estimate the effectiveness and safety of this combination treatment for GBM patients with LMD.

\section{Methods}

This study was approved by the Medical Ethics Committee of Beijing Tiantan Hospital, Capital Medical University. Between January 2012 and December 2019, intrathecal MTX combination with systemic chemotherapy was administered to $26 \mathrm{GBM}$ patients (age range, 18-61 years old; median, 43 years old) with LMD in our institution. GBM diagnosis was confirmed by specialized neuro-pathologists according to the 2016 WHO classification of brain tumors.

Diagnosis of LMD involves enhanced MRI and/or positive morphology of CSF cells. Concomitant chemoradiotherapy (CCRT) adjuvant TMZ chemotherapy was performed according to the standard protocol[1, 2].

Data collected for each patient included sex, date of birth, date of initial glioma diagnosis, date of LMD diagnosis, Karnofsky Performance Status (KPS) score at LMD diagnosis, molecular pathologic analysis, initial and subsequent LMD treatments, first CSF results after LMD diagnosis, hematological toxicity of treatment, and date of death or last follow-up.

Treatment plan: Each treatment cycle was 28 days; b). MTX was intrathecally injected 2-3 times in each cycle, with a single dose of $10 \mathrm{mg}$ once a week; c). TMZ regiment was the first choice when 6 cycles of TMZ systemic chemotherapy had not been completed (TMZ 150-200 mg/m²/d), 5 days every 28 days; d). Etoposide and carboplatin(EC) regimen was used in patients who failed in the previous TMZ regiment less than 6 months. Carboplatin AUC5 was administered day 1 every 28 days, and etoposide was administered at $100 \mathrm{mg} / \mathrm{m}^{2} / \mathrm{d}$, day $1-3$ every 28 days; e). TMZ combined with cisplatin(TP) regimen was used in patients whose previous TMZ regimen of over 6 months failed. Cisplatin was administered at 30 $\mathrm{mg} / \mathrm{m}^{2} / \mathrm{d}$, day $1-3$ every 28 days, and TMZ was administered at $150-200 \mathrm{mg} / \mathrm{m}^{2} / \mathrm{d}$, day $1-5$, every 28 
days; f). Bevacizumab (BEV) was administered to patients with severe brain edema after showing poor reaction to conventional dehydration treatment. BEV was administered at $5 \mathrm{mg} / \mathrm{kg}$ day 1 , every 28 days; g). Chemotherapy dose and interval were adjusted according to chemotherapy principles; h). MRI and/or CSF morphology were reviewed every 2 months. If the treatments were evaluated as effective, then they were continued for no more than eight cycles. If invalid, then the treatment is changed to another combined chemotherapy regimen until the tumor progresses, the patient gives up treatment, or dies.

\section{Statistical analysis}

The overall survival, time from initial GBM diagnosis to death, time from GBM diagnosis to LMD diagnosis, and time from LMD diagnosis to death or last follow-up (1 January 2021) were evaluated using the Kaplan-Meier method. The comparison between patients' characteristics was assessed using log-rank test. Univariate Cox regression models were applied to assess the effect of covariates of interest on time-to-event endpoint. A p value of $<0.05$ was considered significant for all analyses. All computations were carried out in SPSS 23.0.

\section{Results}

Twenty-six GBM patients developed LMD and were treated in our institution.

\section{Patient's characteristics before LMD diagnosis}

Among the 26 patients included in the analysis, 16 (61.5\%) were men and 10 (38.5\%) were women, with a median age of 43 years old (range 16-61 years old). Most patients' primary tumor location were supratentorial, and only two occurred in the infratentorial (cerebellum). Seven patients' tumors $(26.9 \%)$ had tumor gross total resection. Eighteen tumors (69.2\%) within $1 \mathrm{~cm}$ of the ventricular system or the ventricular system were open during initial surgery. All the patients have molecular pathology, which was tested by immunohistochemistry or next generation sequencing. The patterns of treatment after GBM diagnosis and before LMD diagnosis are provided in detail in Table 1.

\section{Patient's characteristics at time of LMD diagnosis}

Twenty-six of these LMD patients have four types of clinical symptoms, namely, headache (46.2\%), backache $(15.4 \%)$, lower extremity weakness $(11.5 \%)$, and visual changes $(3.8 \%)$. Only six patients $(23.1 \%)$ were asymptomatic at diagnosis, which was performed by routine imaging examination. With the development of the disease, most patients appeared to have intracranial hypertension syndrome, severe headache, progressive cognitive impairment, cranial nerve damage, ataxia, and other brain and spinal cord injury symptoms. The details are presented in Table 2.

The median time from GBM surgery to diagnosis of LMD was 9.4 months (range, 0.7-41.4 months). One patient (3.8\%) was diagnosed with LMD (spinal cord metastasis) at the time of initial GBM diagnosis, and the remaining 25 patients were diagnosed after surgery. 
All 26 patients had positive MRI findings. Leptomeningeal tumor enhancement was found in the brain around the contours of the gyri and sulci or multiple nodular deposits in the subarachnoid space, cerebellar folia, and the cortical surface. When these occurred in the spinal cord, linear or nodular enhancement along the surface can be conclusive features of LMD.

All 26 patients underwent a single lumbar puncture for the purpose of CSF analysis, but the cytologic examination of only half of them $(13,50 \%)$ showed positive for malignant cells. Twenty-one patients $(80.8 \%)$ had total protein levels at above the normal range in the CSF.

For the convenience of intrathecal chemotherapy and to avoid lumbar puncture-related metastasis, 20 patients $(76.9 \%)$ accepted Ommaya reservoir implantation. According to the chemotherapy principle mentioned above, five patients used TMZ as systemic chemotherapy, eight accepted TMZ+DDP (TP), and 13 were treated with vp-16+CBP $(E C)$. The median of chemotherapy cycles was 4 (range 1-8). Four patients had systemic chemotherapy plan alternated after first line failure. One patient accepted vemurafenib therapy because of BRAF mutation.

\section{Toxicity}

Significant treatment-related side effects are gastrointestinal toxicity and myelotoxicity. According to Common Terminology Criteria of Adverse Events version 4.03, 24 patients (92.3\%) had a grade 1-2 gastrointestinal side effects, whereas 19 patients $(73.1 \%)$ had grade $1-2$ myelotoxicity.

\section{Survival}

As of the last follow-up, six patients were still alive. The median overall survival for all patients from glioblastoma diagnosis was 27.8 months. Median survival time from diagnosis of glioblastoma to LMD was 9.4 months (range 2-59 months). Median survival from the diagnosis of LMD was 10.5 months (Figure 1A). Ten patients showed improvement in neurological symptoms and imaging. The imaging of typical cases is shown in Figure 2. Eight patients had stable disease, whereas treatment was not effective in the remaining eight patients.

Univariate analysis showed that median OS from the diagnosis of LMD was significantly different between KPS $₫ 60$ and KPS $\leq 60$ (16 months vs. 9 months $p=0.002)$, Ommaya reservoir implantation or not (15 months vs. 6 months $\mathrm{p} \otimes 0.001$ ), gross total resection of tumor or not(median $24.7,95 \% \mathrm{Cl}[15.1,34.3]$ vs. $10.9,95 \% \mathrm{Cl}[8.0,13.7], p=0.022)$ (Figure $1 B, C, D)$. MGMT methylation $(p=0.187)$, communicating with the ventricle at time of GBM diagnosis $(p=0.778)$, total protein in CSF $(p=0.321)$, and BEV use $(p=0.085)$ have no significant outcome association (Table 3).

Multivariate analysis showed that longer overall survival from diagnosis of LMD was associated with KPS $₫ 60(p=0.037)$ and Ommaya reservoir implantation $(p=0.014)$ (Table 4).

Table 1 Clinical characteristics of the patients at time of GBM diagnosis 
Table 2 Patients characteristics of LMD diagnosis

Table 3:Univariate cox regression models-from LMD to death according to treatment

Table 4:Multivariate cox regression models-from LMD to death according to treatment

Figure 1 Kaplan Meier survival curve from LMD diagnosis according to treatment. A. OS of all LMD patients; B. OS was significantly different whether gross total resection of tumor or not(median 24.7, $95 \% \mathrm{Cl}[15.1,34.3]$ vs. $10.9,95 \% \mathrm{Cl}[8.0,13.7], p=0.022)$. C. Difference between $\mathrm{KPS} \varangle 60$ and $\mathrm{KPS} \leq 60(16$ months vs. 9 months $p=0.002)$; D. Difference between Ommaya reservoir implantation or not (15 months vs. 6 months $\mathrm{p} \llbracket 0.001)$.

Figure 2. MRI scans ( $\mathrm{T} 1+$ Gadolinium) of patient before (upper panel) and after 4 cycles combination therapy(lower panel) is shown. The lesion in the right temporal lobe is stable after surgery. The multiple lesions in the cervical(middle column, red arrows) and lumbar(right column, red arrows) spine obviously remitted.

\section{Discussion}

LMD in GBM patients is a serious complication with a worse outcome. There is no consensus on the treatment for LDM. Disease progression or treatment-related complications, such as intrathecal treatment or bleeding and infection after ventricular-abdominal shunt, can sometimes lead to fatal results[6, 12]. Considering the multifocal nature of LMD, surgical treatment is not appropriate. Palliative radiotherapy is the most commonly used treatment that can relieve symptoms and slightly improves survival[13, 14]. Completed clinical trials have explored the application of a variety of single-use intrathecal chemotherapeutics, including topotecan, methotrexate, and cytarabine, to LMD. Although the safety evaluation is satisfactory, none of the single-use drugs can significantly improve the survival rate of LMD patients[15]. Single-use BEV or in combination with irinotecan showed inconsistent clinical benefits[4, 16, 17]. Targeted therapy can be used in selected cases with sensitive mutation, but it is not widely used due to the insufficient detection or the less sensitive mutation of glioma itself[18-20]. Although Chimeric Antigen Receptor T-Cell Immunotherapy therapy for IDH wild-type MGMT methylated GBM combined with LMD has encouraging effects and no related side effects[21], it is difficult to find a suitable target. Immunosuppressive microenvironment and subsequent toxicity still limit immunotherapy.

Chemotherapy is one of the main brain tumor treatment methods. Multiple chemotherapeutic regimens have been investigated, such as different drugs, i.e., single-use or combination use (TMZ, lomustine, irinotecan, and BEV) $[15,16]$. No significant effect was achieved from the intrathecal injection chemotherapy of different drugs (methotrexate or cytarabine)[5,22]. Based on our team's previous research work on the use of methotrexate in the treatment of gliomas and on the combined therapeutic effect of chemotherapy on recurrent gliomas, we combined MTX intrathecal injection and systemic chemotherapy. The results showed that the current OS improved compared with past OS[4-6]. As far as we know, this study is a clinical study with the largest number of samples in the treatment of GBM 
combined with LMD by intrathecal MTX combined with systemic chemotherapy, and has achieved good clinical research conclusions.

Previous studies have shown that MGMT promoter methylation status can be used as an indicator of prognosis of the newly diagnosed GBM patients. It was also proposed as a risk factor of LMD development in glioma patients[23]. The suspected mechanisms include the increase in the survival of patients subjected to MGMT methylation treatment. MGMT status has no correlation with OS for GBM after LMD diagnosis. Therefore, for patients diagnosed with LMD, MGMT methylation status does not determine whether TMZ can be used or not.

BEV has been suggested as a promoter of LMD[17], but available data are conflicting. Considering the high price and the fact that BEV was not approved by FDA in China until this year, all of the patients in this cohort did not use BEV when the GBM was first diagnosed. We only used BEV in patients with severe brain edema and who did not respond to conventional dehydration. The results showed that BEV use had a negative effect on the OS of patients with LMD but played a positive role in relieving intracranial hypertension.

Some studies have shown that ventricle opening during surgery or tumor invasion to ventricle system may be one of the main factors causing $\operatorname{LMD}[7,24]$. In our study, 18 patients with LMD $(69.2 \%)$ showed communication with the ventricle at GBM diagnosis, and this result is similar to that obtained in other studies. When it comes to the relationship between ventricles opening and OS of LMD patients, there was no significant difference between the two groups, possibly because of the small sample sizes. Further verification is needed.

In this study, the MRI imaging abnormalities of the brain and spinal cord were regarded as LMD diagnosis criteria, and only 13 patients showed positive results in the CSF morphological examination. Generally, the CSF morphology test should comprise more than one test and should be combined with CSF flow cytometry if necessary. Considering that our CSF morphology result is a single lumbar puncture test before MTX intrathecal injection, the presence of false negatives is possible.

The CSF protein content is a clinical characteristic of patients with brain tumor. Twenty-one of 23 patients showed increased levels of CSF protein in this study, and the protein content of CSF decreased in effectively treated patients. Therefore, we supposed that CSF protein content can be used as a marker for disease diagnosis and treatment efficiency evaluation.

Our results showed that total resection of brain tumor at initial diagnosis and KPS $₫ 60$ at the time of diagnosis of LMD are good prognostic factors, and this conclusion is similar to those of other studies[4].

Ommaya reservoir implantation has been widely used in the treatment of LDM of different cancers. It can avoid the injury caused by lumbar puncture and the corresponding risks. In this study, Ommaya reservoir implantation was a positive factor for the OS of LMD patients. In addition to MTX intrathecal 
administration, we also used Ommaya reservoir as a simple device for external ventricular drainage at the end stage of the disease, which can sometimes alleviate the symptoms of high intracranial pressure.

In the study, some patients showed improvement in clinical symptoms and partial remission of imaging. Considering the lack of a unified evaluation standard, it is impossible to evaluate the curative effect. Therefore, this study only takes OS as the main end point and evaluation standard of the curative effect.

\section{Limitation}

Limitations of our study include its single-center and retrospective nature. It is a one arm study that lacks a control group. The sample size is insufficient, and the conclusion needs further verification. Our study also did not permit the assessment of quality of life and morbidity. We have launched a single-center prospective study to further verify the efficacy of MTX combined with systemic chemotherapy in the treatment of GBM with LMD.

\section{Conclusion}

LMD is a lethal outcome of glioma patients with an increasing incidence rate. It remarkably shortens patients' OS. Intrathecal methotrexate combined with systemic chemotherapy is an effective therapy for GBM patients with LMD. KPS $₫ 60$, gross resection of brain tumor, and Ommaya reservoir implantation are positive prognostic factors for patients with LMD.

\section{Abbreviations}




\begin{tabular}{|ll|}
\hline BEV & Bevacizumab \\
\hline CBP & Carboplatin \\
\hline CCRT & Concomitant chemoradiotherapy \\
\hline CSF & Cerebrospinal fluid \\
\hline DDP & Cisplatin \\
\hline GBM & Glioblastoma \\
\hline KPS & Karnofsky Performance Status \\
\hline IDH & Isocitrate dehydrogenase \\
\hline LMD & Leptomeningeal spread of disease \\
\hline MGMT & O6-methylguanine-DNA methyltransferase \\
\hline MRI & Magnetic resonance imaging \\
\hline MTX & Methotrexate \\
\hline OS & Overall survival \\
\hline TERT & Telomerase reverse transcriptase \\
\hline TMZ & Temozolomide \\
\hline TTF & Tumor-treating fields \\
\hline
\end{tabular}

\section{Declarations}

Funding

This work was supported by the National Natural Science Foundation of China (No.81972338).

\section{Acknowledgements}

The authors sincerely thank all the patients and their families for their participation in the present study.

\section{Availability of data and materials}

All data generated or analyzed during this study are included in this published article.

\section{Authors' contributions}

KX, L W-B designed and performed this study; CF, KZ, WC, Q Z-H, LY, LS, C J-X, LY, LP and JB contributed to the patient recruitment and collected the data; $Y$ S-B, $L H$, and $W Y$ - L performed the statistical analysis; $P$ $\mathrm{Y}-\mathrm{C}, \mathrm{W} \mathrm{X}-\mathrm{M}$ and $\mathrm{F} \mathrm{A}-\mathrm{Z}$ help wrote the manuscript. All authors read and approved the final manuscript. 


\section{Competing interests}

The authors declare no potential conflicts of interest with respect to the research, authorship, and/or publication of this article.

\section{Consent for publication}

Written informed consent was obtained from all patients.

\section{Ethics approval and consent to participate}

Written informed consent was obtained from each patient at the time of enrollment. The study was carried out in accordance with the Declaration of Helsinki on experimentations involving human subjects, was approved by the Institutional Ethics Review Boards of Beijing Tiantan Hospital.

\section{Author details}

1 Department of Neuro-oncology, Cancer Center, Beijing Tiantan Hospital, Capital Medical University, 119 South Fourth Ring West Road, Fengtai District, Beijing, 100070, China.2 Department of Neurosurgery, Beijing Tiantan Hospital, Capital Medical University, 119 South Fourth Ring West Road, Fengtai District, Beijing, 100070, China.3 Department of Oncology, Beijing Shijitan Hospital, Capital Medical University, 10 Tieyi Road, Haidian District, Beijing, 100038, China. 4 Clinical Medicine, Shanghai Jiao Tong University School of Medicine, 227 Chongqing South Road, Huangpu District, Shanghai, 200025, China.

\section{References}

1. Stupp, R., et al., Radiotherapy plus concomitant and adjuvant temozolomide for glioblastoma. N Engl J Med, 2005. 352(10): p. 987-96.

2. Stupp, R., et al., Chemoradiotherapy in malignant glioma: standard of care and future directions. J Clin Oncol, 2007. 25(26): p. 4127-36.

3. Stupp, R., et al., Effect of Tumor-Treating Fields Plus Maintenance Temozolomide vs Maintenance Temozolomide Alone on Survival in Patients With Glioblastoma: A Randomized Clinical Trial. JAMA, 2017. 318(23): p. 2306-2316.

4. Mandel, J.J., et al., Leptomeningeal dissemination in glioblastoma; an inspection of risk factors, treatment, and outcomes at a single institution. J Neurooncol, 2014. 120(3): p. 597-605.

5. Noh, J.H., et al., Optimal treatment of leptomeningeal spread in glioblastoma: analysis of risk factors and outcome. Acta Neurochir (Wien), 2015. 157(4): p. 569-76. 
6. Autran, D., et al., Leptomeningeal Gliomatosis: A Single Institution Study of 31 Patients. Anticancer Res, 2019. 39(2): p. 1035-1041.

7. Alatakis, S., G.M. Malham and C. Thien, Spinal leptomeningeal metastasis from cerebral glioblastoma multiforme presenting with radicular pain: case report and literature review. Surg Neurol, 2001. 56(1): p. 33-7; discussion 37-8.

8. Krause, M.Z., et al., 5-ALA-Induced Fluorescence in Leptomeningeal Dissemination of Spinal Malignant Glioma. World Neurosurg, 2018. 110: p. 345-348.

9. Narayana, A., et al., Feasibility of using bevacizumab with radiation therapy and temozolomide in newly diagnosed high-grade glioma. Int J Radiat Oncol Biol Phys, 2008. 72(2): p. 383-9.

10. de Groot, J.F., et al., Tumor invasion after treatment of glioblastoma with bevacizumab: radiographic and pathologic correlation in humans and mice. Neuro Oncol, 2010. 12(3): p. 233-42.

11. Bai Y, Zhong XS, Li WB. Glioblastoma progression is inhibited by methotrexate via RAS/MEK/ERK/MYC/CD47 signaling pathways. Electron J Metab Nutr Cancer, 2018; 5(2): p.144-150.

12. Saito, R., et al., Fatal hemorrhage after radiochemotherapy for leptomeningeal dissemination of glioma: report of two cases. Surg Neurol, 2002. 57(1): p. 46-8.

13. Karaca, M., et al., Cases of glioblastoma multiforme metastasizing to spinal cord. Neurol India, 2006. 54(4): p. 428-30.

14. Fiorentino, A., et al., Radiotherapy and bevacizumab for intramedullary and leptomenigeal metastatic glioblastoma: a case report and review of the literature. Int J Neurosci, 2012. 122(11): p. 691-4.

15. Birzu, C., et al., Leptomeningeal Spread in Glioblastoma: Diagnostic and Therapeutic Challenges. Oncologist, 2020. 25(11): p. e1763-e1776.

16. Burger, M.C., et al., Addition of Anti-Angiogenetic Therapy with Bevacizumab to Chemo- and Radiotherapy for Leptomeningeal Metastases in Primary Brain Tumors. PLoS One, 2016. 11(6): p. e0155315.

17. Ruff, M.W. and S.H. Kizilbash, Glioblastoma with bilateral extraocular muscle infiltration preceded by evidence of vascular tropism. J Clin Neurosci, 2019. 61: p. 277-278.

18. Leaver, K.E., et al., Response of metastatic glioma to vemurafenib. Neurooncol Pract, 2016. 3(4): p. 268-271.

19. Burger, M.C., et al., Dabrafenib in patients with recurrent, BRAF V600E mutated malignant glioma and leptomeningeal disease. Oncol Rep, 2017. 38(6): p. 3291-3296. 
20. Woo, P., et al., Regression of BRAF (V600E) mutant adult glioblastoma after primary combined BRAFMEK inhibitor targeted therapy: a report of two cases. Oncotarget, 2019. 10(38): p. 3818-3826.

21. Brown, C.E., et al., Regression of Glioblastoma after Chimeric Antigen Receptor T-Cell Therapy. N Engl J Med, 2016. 375(26): p. 2561-9.

22. Zhao, K.H., et al., Antiglioma effects of cytarabine on leptomeningeal metastasis of high-grade glioma by targeting the PI3K/Akt/mTOR pathway. Drug Des Devel Ther, 2017. 11: p. 1905-1915.

23. Brandes, A.A., et al., Recurrence pattern after temozolomide concomitant with and adjuvant to radiotherapy in newly diagnosed patients with glioblastoma: correlation With MGMT promoter methylation status. J Clin Oncol, 2009. 27(8): p. 1275-9.

24. Grabb, P.A., A.L. Albright and D. Pang, Dissemination of supratentorial malignant gliomas via the cerebrospinal fluid in children. Neurosurgery, 1992. 30(1): p. 64-71.

\section{Tables}

Due to technical limitations, table 1, 2, 3, 4 is only available as a download in the Supplemental Files section.

\section{Figures}


A

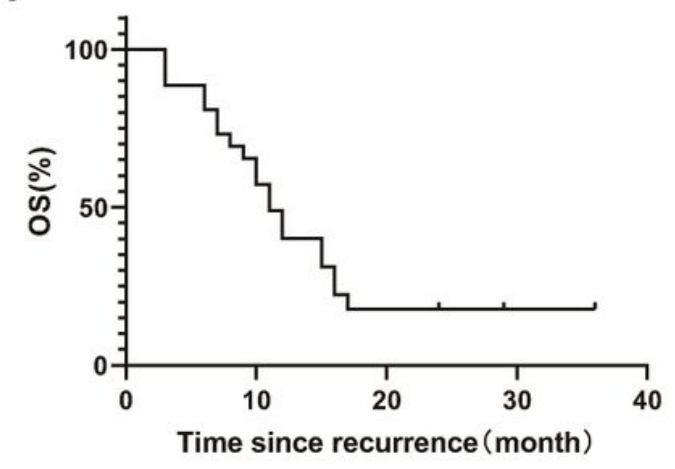

C

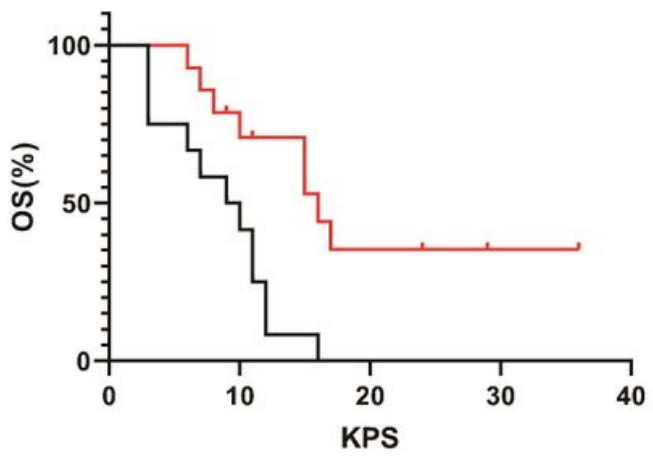

B

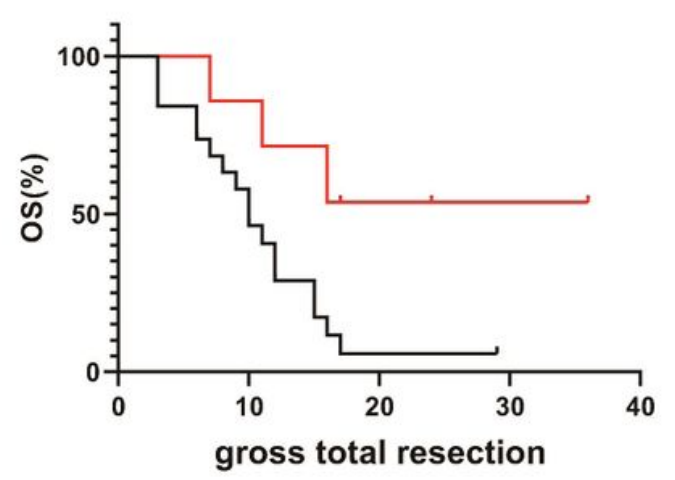

- NO

- YES

$\mathrm{P}=0.022$

D

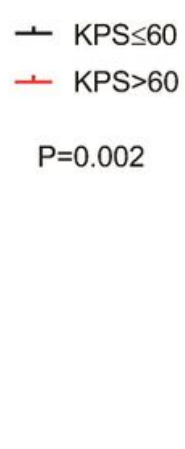

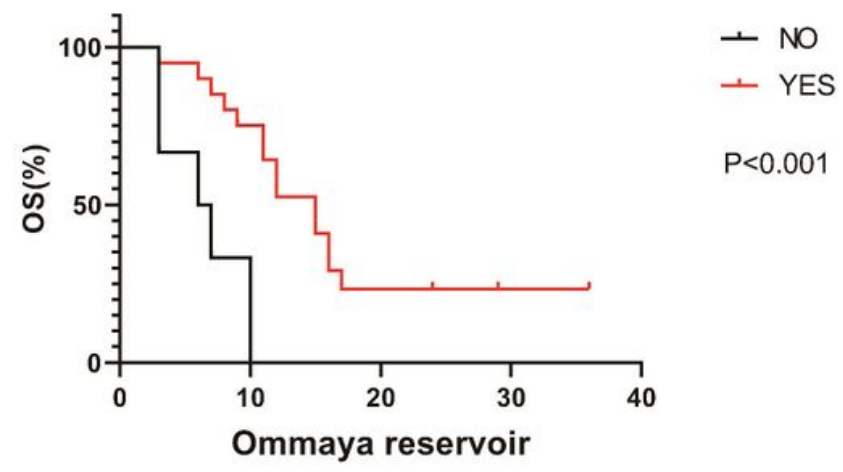

Figure 1

Kaplan Meier survival curve from LMD diagnosis according to treatment. A. OS of all LMD patients; B. OS was significantly different whether gross total resection of tumor or not(median $24.7,95 \% \mathrm{Cl}[15.1,34.3]$ vs. $10.9,95 \% \mathrm{CI}[8.0,13.7], \mathrm{p}=0.022)$. C. Difference between KPS $\otimes 60$ and KPS $\leq 60$ (16 months vs. 9 months

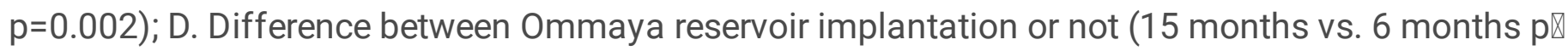
0.001). 


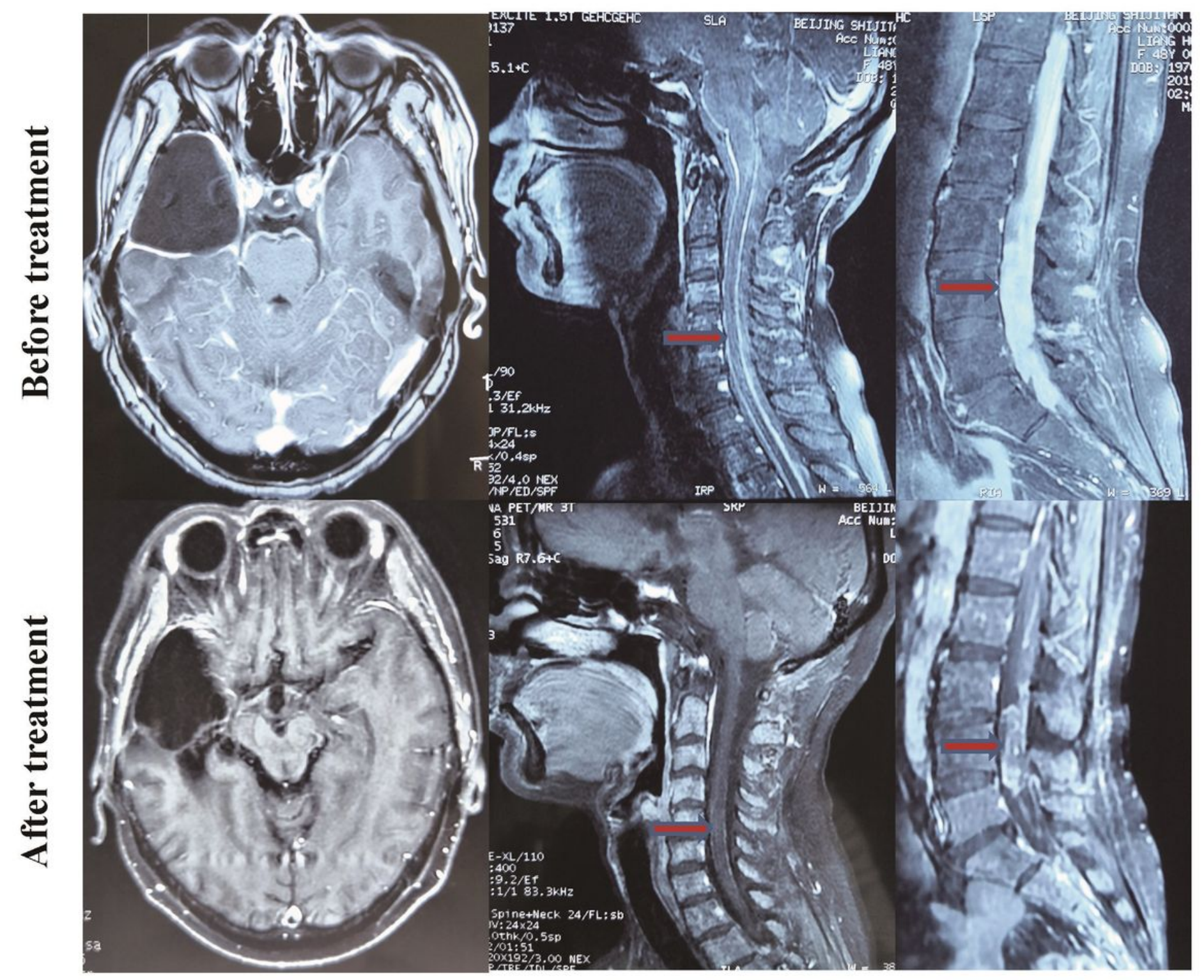

Figure 2

MRI scans ( $T 1+$ Gadolinium) of patient before (upper panel) and after 4 cycles combination therapy(lower panel) is shown. The lesion in the right temporal lobe is stable after surgery. The multiple lesions in the cervical(middle column, red arrows) and lumbar(right column, red arrows) spine obviously remitted.

\section{Supplementary Files}

This is a list of supplementary files associated with this preprint. Click to download.

- Table1.xlsx

- Table2.xlsx

- Table3.xlsx 
- Table4.xIsx

Page 16/16 